\title{
Assessment of CAUDA 70 Score in acute exacerbation of chronic obstructive pulmonary disease
}

\author{
Prasanna Purna ${ }^{1}$, Anil Kumar K., ,", Subhash Bharadwaj Reddy ${ }^{3}$, B. Mohan ${ }^{4}$, C. Rajesh Reddy \\ ${ }^{1}$ Professor \& HOD, ${ }^{2,5}$ Assistant Professor, ${ }^{3,4}$ Post graduate, Dept. of Respiratory Medicine, Narayana Medical College and \\ Hospital, Nellore, Andhra Pradesh, India
}

*Corresponding Author:

Email: anil.kodavala@gmail.com

\begin{abstract}
Background: Physicians lack a robust and validated method of measuring severity or predicting poor outcome in patients with acute exacerbation of COPD (AECOPD). To study the prognosis in COPD patients with acute exacerbation using CAUDA 70 score.

Materials and Methods: 104 patients of age more than 40 years with acute exacerbation of COPD admitted department of respiratory medicine, narayana medical college and hospital, Nellore. CAUDA 70: C: Confusion, Acidosis ( $\mathrm{pH}<7.35)$, Urea $>7 \mathrm{mmol} / \mathrm{L}$, MRC Dyspnoea score $>4$, Albumin $<35 \mathrm{~g} / \mathrm{L}$, Age $>70$ years. One point was assigned to each variable present, giving a six point scoring system. The patients were followed up upto discharge and the outcome of the patients was correlated with the score to analyse prognosis.

Results: 29(27.8\%) patients obtained score upto 2. In these patients, $24(82.75 \%)$ recovered without ventilation, $5(17.24 \%)$ required NIV and no patients required invasive ventilation and there were no deaths in patients with this score. $75(72.2 \%)$ patients obtained scores of 3 to 6 . In these, 48(64\%) patients required NIV, 15(20\%) patients required invasive ventilation, 4 $(5.3 \%)$ patients died and only $8(10.66 \%)$ patients recovered without any ventilation.

Conclusions: CAUDA 70 score proved to be an effective scoring system in predicting the prognosis in Acute Exacerbation of COPD patients.
\end{abstract}

Keywords: Acute Exacerbation of COPD; CAUDA 70 score; Mechanical ventilation.

\section{Introduction}

COPD is characterized by chronic irreversible airway obstruction leading to gradual, progressive decline of lung function punctuated by repeated episodes of exacerbations requiring hospitalization. Chronic Obstructive Pulmonary Disease (COPD) represents a significant burden in terms of morbidity and mortality, accounting for nearly $6 \%$ of deaths globally in 2008[1]. An episode of acute exacerbation of COPD (AECOPD) was defined at the Aspen workshop as "a sustained worsening of the patient's condition from the stable state and beyond normal dayto-day variations that is acute in onset and necessitates a change in regular medication in a patient with underlying COPD[2].

AECOPD can be mild, moderate or severe, so physicians should assess the severity of the exacerbation in order to decide the treatment modality. Acute exacerbation in COPD worsens the symptoms and increases the mortality. Acute exacerbations of COPD (AECOPD) account for one in eight hospital admissions[3]. Early prediction of prognosis in AECOPD is required to decrease the mortality and to improve the quality of life. Clinicians are unable accurately to predict prognosis in patients hospitalised with AECOPD [4]. A majority (up to three-fourths) of episodes of AECOPD are triggered by infections[5]. Environmental pollution is likely to be a trigger in a small proportion of patients (up to 10\%) while in almost a quarter of patients, no cause may be identified[6]. Decreased air temperature and other meteorological factors may contribute to the occurrence of an AECOPD.

Unfortunately there is no widely accepted scoring system in assessing the severity of exacerbation and predicting its prognosis. The scoring system can help in the triage of the patient and classifying them into low risk or high risk.

Current study designed to determine CAUDA 70 score in patients with acute exacerbation COPD admitted in respiratory medicine department in our hospital. In our study the effectiveness of the CAUDA 70 score in predicting need for mechanical ventilation and overall prognosis was tested, because it is simple and rapid score which uses six easily measurable clinical and diagnostic parameters.

COPD not only involves respiratory system but also many other systems. So we cannot assess the severity only on the basis of FEV1 value. To fulfill this need, a different scoring system known as CAUDA 70 is developed.

\section{Materials and Methods}

A prospective observational study consists 104 patients admitted under the Department of Respiratory Medicine, Narayana medical college and hospital, Nellore, Andhra Pradesh, India during January-June 2018.

Inclusion criteria:

1. Age above 40 years 
2. Previously diagnosed as COPD (as per gold guidelines)

3. With a history of smoking or biomass fuel exposure

4. Presented with acute exacerbation symptoms like increased breathlessness, cough, expectoration.

\section{Exclusion criteria:}

1. Age less than 40 years

2. Other pulmonary diseases like asthma, bronchiectasis, active PTB, ILD, pleural effusion etc.

3. Patients with CCF, pulmonary HTN and other cardiac diseases, renal failure, HTN, diabetes.

\section{Procedure:}

All the patients were admitted and following six variables are noted.

1. Confusion

2. Acidosis, $\mathrm{pH}<7.35$

3. Urea $>7$ milli moles per lit

4. Dyspnea grade-IV

5. Albumin $<35 \mathrm{~g} / \mathrm{lit}$

6. Age 70 or above

Each variable was given single point in the score. Thus every patient gets score between 0 to 6 .

Patients were followed till discharge.

Prognosis [outcome] assessed as:

1. Patients recovered without any ventilation,

2. Those requiring noninvasive ventilation;

3. Those requiring invasive ventilation;

4. Death.

Outcome was correlated to the CAUDA 70 score in each patient.

Statistical analysis done by using Statistical Package for the Social Sciences (SPSS) software.

Statistical significance between CAUDA70 score and the prognosis is tested by chi square test. Our study shows the prognosis is correlated to CAUDA70 score with $\mathrm{p}$ value of $0.000(<0.0005)$

\section{Results}

Out of 104 patients $99(95.2 \%)$ are males and 5 (4.8\%) are females.

Of them $20.2 \%$ had confusion.

$81.7 \%$ had acidosis.

$35.6 \%$ had elevated urea.

$64.4 \%$ had grade 4 dyspnoea.

$69.2 \%$ had low albumin.

$48 \%$ had age 70 and above.

Outcome in total 104 patients is as follows.

$51 \%$ (53)- non-invasive ventilation.

$14.4 \%$ (15)- invasive ventilation.

$30.8 \%$ (32)- recovered without any ventilation.

$3.8 \%$ (4)-deaths.

This outcome correlated to CAUDA 70 score:

$29(27.8 \%)$ out of 104 patients obtained scores up to 2 .

In score up to $282.75 \%$ (24) recovered without ventilation.

$17.24 \%$ (5) required non -invasive ventilation.
No patient required invasive ventilation and no deaths occurred with the score upto 2

$75(72.2 \%)$ patients out of 104 in our study obtained score 3 to 6 .

Only $10.66 \%(8)$ of them recovered without any ventilation.

$64 \%$ (48) patients required non -invasive ventilation.

$20 \%$ (15) patients required invasive ventilation.

$5.33 \%$ (4) deaths occurred with score 3 to 6 in the study.

Our study shows the prognosis is correlated to CAUDA70 score with p value of $0.000(<0.0005)$

All the parameters individually have statistical significance with prognosis in AE COPD.

\section{Discussion}

COPD symptoms (breathlessness, cough) worsen due to acute exacerbation (i.e.AE COPD) which occur due to bacterial or viral infections.

The principal result of the present study is the identification of strong predictors of in-hospital mortality in patients admitted with AECOPD. The study allowed dervation of scoring system based on these factors. To our knowledge, this prediction tool has the highest level of discrimination of any scoring system yet produced for prediction of in-hospital mortality in AECOPD.

In AE COPD there is increased mortality. Only a small number of studies have previously derived predictive scoring systems for in hospital mortality in AECOPD. None of these studies have been independently validated, subject to an impact analysis or implemented into clinical practice[6-9].

It is useful to consider possible reasons why the six variables in the new prediction score correlate with in-hospital mortality in AECOPD. Urea has previously appeared as an important predictor of poor outcome in respiratory disease, reflect acute kidney injury resulting from volume depletion due to hyperventilation[8-13]. Confusion, marker of poor outcome in community acquired pneumonia, arise in AECOPD due to hypercapnia.

Patients with a score of 0-1 are at low risk of death and are likely to be safely managed at home. Patients $\mathrm{w}$ ith a score of 2 are also at low risk but will need to be $h$ ospitalised if they are confusedor acidotic. Scores of $3 \mathrm{o}$ $\mathrm{r}$ more indicate that a patient is at high risk of in-hospita 1 mortality, asthe mortality at this level climbs to $14 \%$.

Results of our study show that patients with CAUDA 70 score 3 and above have poor prognosis compared to those having score of 0 to 2 .

In our study, 75(72.25\%) are having score 3 to 6 in them $64 \%(48)$ required non- invasive ventilation, $20 \%$ (15) required invasive ventilation, only $10.66 \%$ (8) recovered without ventilation and $5.2 \%$ (4) deaths occurred.

$29(27.8 \%)$ of total 104 patients have score up to 2 . Of them, 5 patients $(17.24 \%)$ only required non- 
invasive ventilation and $82.7 \%$ (24) patients recovered without any ventilation.

From the above results it is clear that most of the patients with 3 and above score required invasive (20\%); non-invasive ventilation $(64 \%)$.

All 4 deaths of the study occurred with score 3 to 6.

$10 \%$ of the patients in this group recovered without ventilation.

In CAUDA70 score up to 2 patients, $83 \%$ recovered without ventilation, $17 \%$ required NIV, no patient required invasive ventilation and no deaths.

All the parameters individually have statistical significance with prognosis in AE COPD.

Of the 6 parameters, acidosis is present in $81.7 \%$ followed by albumin $69.2 \%$, grade 4 breathlessness in $64.4 \%$ and age 70 and above in $48 \%$. Urea was raised in $35.6 \%$ and confusion was present in $20 \%$.

Acidosis, grade 4 breathlessness indicate respiratory failure and confusion indicate $\mathrm{CO}_{2}$ retention. Low albumin and raised urea indicate systemic involvement of AE COPD. Thus all the parameters suggest the severity of AECOPD and the presence of respiratory failure. So high CAUDA 70 score resulted in poor prognosis in $\mathrm{AE}$ COPD.

This more complex scoring system performed sligh tly better than the chosen scoring system, with an AUC of 0.85 (as opposed to 0.84 ).

However, despite its slightly increased level of disc rimination and a slightly lower mortality rate at low pre diction scores, these were not deemed to be significant enough to outweigh the ease of use of the chosen single-point scoring system.

CAUDA 70 score is developed in U.K by EXODUS study (exacerbation of obstructive lung disease managed in U.K secondary care) between 2009 and 2012.

In 2015 in Egypt “ assessment of in hospital mortality and need for mechanical ventilation in AECOPD 2 year prospective study" they compared 4 scoring systems CAUDA 70, BAP 65, CURB 65, CAPS. Of the four, CAUDA 70 score gave good results in predicting need for mechanical ventilation and mortality. They proved that CAUDA 70 score 3 and above have high mortality and recommended the need for mechanical ventilation[12-14].

Our study also shown that AECOPD patient with 3 and above score had increased requirement of mechanical ventilation and higher mortality.

\section{Conclusions}

To assess the prognosis in AECOPD, CAUDA 70 score proved to be simple and effective method with six easily observable clinical variables. Based on the score we can predict the need for mechanical ventilation and prevent mortality. These findings should help in determining which patients are at high risk of inhospital death, and whether they should be managed as an in-patient or at home with early supported discharge. This scoring system also useful in decisions on patient admission, discharge management and health care resource allocation.

\section{Acknowledgement}

We acknowledge the teaching/non-teaching staff for assisting in data record, analysis and interpretation. We acknowledge the scientific staff Dr. Mahaboob V Shaik for preparation of the manuscript.

Conflicts of interest: All authors declared no conflicts of interest.

\section{References}

1. Mathers CD, Loncar D. Projections of global mortality and burden of disease from 2002 to 2030. PLoS Med 2006;3(11):e442.

2. Rodriguez-Roisin R. Towards a consensus definition for COPD exacerbations. Chest. 2000;117:398s-401s.

3. Healthcare Commission. Clearing the air: A national study of chronic obstructive pulmonary disease. London: Commission for Healthcare Audit and Inspection. 2006.

4. Wildman MJ, Sanderson C, Groves J, Reeves BC, Ayres J, Harrison D, Young D, Rowan K. Predicting mortality for patients with exacerbations of COPD and Asthma in the COPD and Asthma Outcome Study (CAOS). QJM: International J Med 2009;102(6):389-99.

5. Ball P. Epidemiology and treatment of chronic bronchitis and its exacerbations. Chest 1995;108:43s-52s.

6. Sunyer J, Saez M, Murillo C, Castellsague J, Martínez F, Antó JM. Air pollution and emergency room admission for chronic obstructive pulmonary disease. Am J Epidemiol 1993;137:701-5.

7. Roche N, Zureik M, Soussan D, Neukirch F, Perrotin D. Predictors of outcomesin COPD exacerbation cases presenting to the emergency departme nt. Eur Respir J 2008;32:953-961.

8. Tabak Y, Sun X, Johannes R, Gupta V, Shorr A. Mortalit $\mathrm{y}$ and Need for Mechanical Ventilation in Acute Exacerbations of Chronic Obstructive Pulmonar y Disease. Arch Int Med 2009;169(17):1595-602.

9. Wildman MJ, Harrison DA, Welch CA, Sanderson C. A new measure of acute physiological derangement for patients with exacerbations of obstructive airways disease: the COPD and Asthma Physiology Score. Respirat Med 2007;101(9):1994-2002.

10. Mohan A, Bhatt S, Mohan C, S. Arora S, Luqman-Arafath T, Guleria R. Derivationof a Prognostic Equation to Predict In-Hospital Mortality and Requirement of Invasive Mechanical Ventilation in Patients with Acute Exacerbation of Chronic Obstructive Pulmonary Disease. Indian J Chest Dis Allied Sci 2008;50:335-42.

11. Lim WS, van der Eerden M, Laing R, Boersma W, Karal us N, Town G, Lewis S, Macfarlane J. Defining community acquired pneumonia severity on presentation to hospital: an international derivation and validation study. Thorax 2003;58:377-82.

12. Archibald R, Chalmers J, Fardon T, Short P, Williamson P, Taylor J, Singanayagam A, Peet L, Al-Khairalla M, Schembri S. Prediction of In-Hospital Mortality in Acute Exacerbations of COPD. Scottish Univ Med J 2012;1(2).

13. Elsokkary R, Ghanem M, Metwally M, Abdelaleem N. Assessment of In-Hospital Mortality and the Need for Mechanical Ventilation in Acute Exacerbations of COPD: 
A 2-Years Prospective In-Hospital Observational Study. Chest 2015;148(4):756A.

14. Celli BR, Cote CG, Marin JM, Casanova C, Montes de Oca M, Mendez RA, Pinto Plata V, Cabral HJ. The bodymass index, airflow obstruction, dyspnea, and exercise capacity index in chronic obstructive pulmonary disease. New England Journal of Medicine. $N$ Engl J Med 2004;350:1005-12.
How to cite this article: Purna P, Anil K.K, Reddy S. B, Mohan B., Reddy C. R. Assessment of CAUDA 70 Score in acute exacerbation of chronic obstructive pulmonary disease. Indian $\mathbf{J}$ Immunol Respir Med. 2018;3(4):170-173. 